\title{
The Effect of Scale on the Applicability of Taylor's Frozen Turbulence Hypothesis in the Atmospheric Boundary Layer
}

\author{
Chad W. Higgins - Martin Froidevaux • \\ Valentin Simeonov • Nikki Vercauteren • Caitlin Barry • \\ Marc B. Parlange
}

Received: 6 June 2011 / Accepted: 16 January 2012 / Published online: 10 February 2012

(C) The Author(s) 2012. This article is published with open access at Springerlink.com

\begin{abstract}
Taylor's frozen turbulence hypothesis is the central assumption invoked in most experiments designed to investigate turbulence physics with time resolving sensors. It is also frequently used in theoretical discussions when linking Lagrangian to Eulerian flow formalisms. In this work we seek to quantify the effectiveness of Taylor's hypothesis on the field scale using water vapour as a passive tracer. A horizontally orientated Raman lidar is used to capture the humidity field in space and time above an agricultural region in Switzerland. High resolution wind speed and direction measurements are conducted simultaneously allowing for a direct test of Taylor's hypothesis at the field scale. Through a wavelet decomposition of the lidar humidity measurements we show that the scale of turbulent motions has a strong influence on the applicability of Taylor's hypothesis. This dependency on scale is explained through the use of dimensional analysis. We identify a 'persistency scale' that can be used to quantify the effectiveness of Taylor's hypothesis, and present the accuracy of the hypothesis as a function of this non-dimensional length scale. These results are further investigated and verified through the use of large-eddy simulations.
\end{abstract}

Keywords Atmospheric boundary layer · Humidity · Raman lidar · Taylor's frozen turbulence hypothesis

\section{Introduction}

The Taylor frozen turbulence hypothesis (Taylor 1938) is universally prevalent in the investigation of fluid flow physics, as it is far more practical to deploy time-resolving instruments to track the temporal evolution of the fluid flow. Reliance on Taylor's hypothesis has diminished

C. W. Higgins $(\bowtie)$

Department of Biological and Ecological Engineering, Oregon State University, Corvallis, OR, USA

e-mail: chad.higgins@oregonstate.edu

M. Froidevaux · V. Simeonov · N. Vercauteren · C. Barry $\cdot$ M. B. Parlange

School of Architecture, Civil and Environmental Engineering, Ecole Polytechnique Federale de Lausanne, 1015 Lausanne, Switzerland 
in the past decade in small-scale experiments due to advancements in particle image velocimetry (PIV) and other laser-based measurement technologies. For field scale measurements, one must still invoke a frozen turbulence assumption, for example in recent analyses of subgrid-scale physics for large-eddy simulation (LES) (Tong et al. 1999; Porté-Agel et al. 1998, 2000a,b, 2001a,b; Higgins et al. 2003, 2004, 2009; Kleissl et al. 2003, 2004; Kelly et al. 2009; Bou-Zeid et al. 2010; Patton et al. 2011). Due to its widespread use, Taylor's hypothesis has been studied theoretically (Lumley 1965; Wyngaard and Clifford 1977; Hill 1996), with simulations (Horst et al. 2004; Dosio et al. 2005; Bahraminasab et al. 2008; Del Alamo and Jimenez 2009; Moin 2009), with laboratory experiments (Willis and Deardorff 1976; Dahm and Southerland 1997), and consequences of Taylor's hypothesis have been inferred from field measurements (Tong 1996; Thomas 2011) but to our knowledge there has been no test of Taylor's hypothesis across the wide range of scales relevant to field measurements. In this paper we describe the Turbulent Atmospheric Boundary-layer, Lidar and Evaporation (TABLE) experiment that was designed, in part, to provide an appropriate test of Taylor's hypothesis.

Formally, Taylor's hypothesis assumes that the advection velocity of the turbulence is much greater than the velocity scale of the turbulence itself. To quote the original statement in Taylor (1938): "If the velocity of the air stream which carries the eddies is very much greater than the turbulence velocity, one may assume that the sequence of changes in $u$ at a fixed point are simply due to the passage of an unchanging pattern of turbulent motion over the point." Invoking this assumption, time is replaced with a distance scaled by velocity,

$$
u(t)=u\left(\frac{x}{U}\right)
$$

where $U$ is the advection velocity, and $x$ and $t$ are distance and time, respectively. Of course Eq. 1 is not strictly true for all time; nonetheless it is reasonable to expect that it will hold if $t$ is sufficiently short. The question then becomes: over what time interval is Eq. 1 generally valid? To quantify the validity of the hypothesis we use the following generalized space-time correlation function following Pope (2000) written in the mean flow direction,

$$
\Phi(\delta x, \delta t)=\frac{\overline{u^{\prime}(x, t) u^{\prime}(x+\delta x, t-\delta t)}}{\sqrt{\overline{u^{\prime}(x, t)^{2}}} \sqrt{\overline{u^{\prime}(x+\delta x, t-\delta t)^{2}}}}
$$

where, the overbar represents averaging in both space and time. Using Eq. 2 we compute the spatial correlation function (in the case of $\delta t=0$ ), the time correlation function (in the case of $\delta x=0$ ) and all cross-correlations in space-time. In this equation, Taylor's hypothesis is represented as the special case $\delta x / \delta t=U$, which follows directly from (1). Therefore, if Taylor's hypothesis is applicable, we would expect high correlation when $\delta x / \delta t=U$. Given a sufficient dataset, one can directly compute $\Phi(\delta x, \delta t)$, set a threshold for an acceptable value of correlation to accept Taylor's hypothesis, and deduce the time over which Taylor's hypothesis is valid using Eq. 1. The difficulty, of course, in such an analysis is acquiring a dataset where $u$ is measured in both space and time simultaneously. In the present study we present a dataset obtained with a Raman lidar suitable for the analysis explained above, and then use a wavelet approach to investigate the importance of eddy size on the applicability of Taylor's hypothesis. Large-eddy simulation results are also used in a comparative analysis. We finally define a persistency scale that is used to then determine the absolute best correlation that can be achieved in Eq. 2 from the data and from LES. 


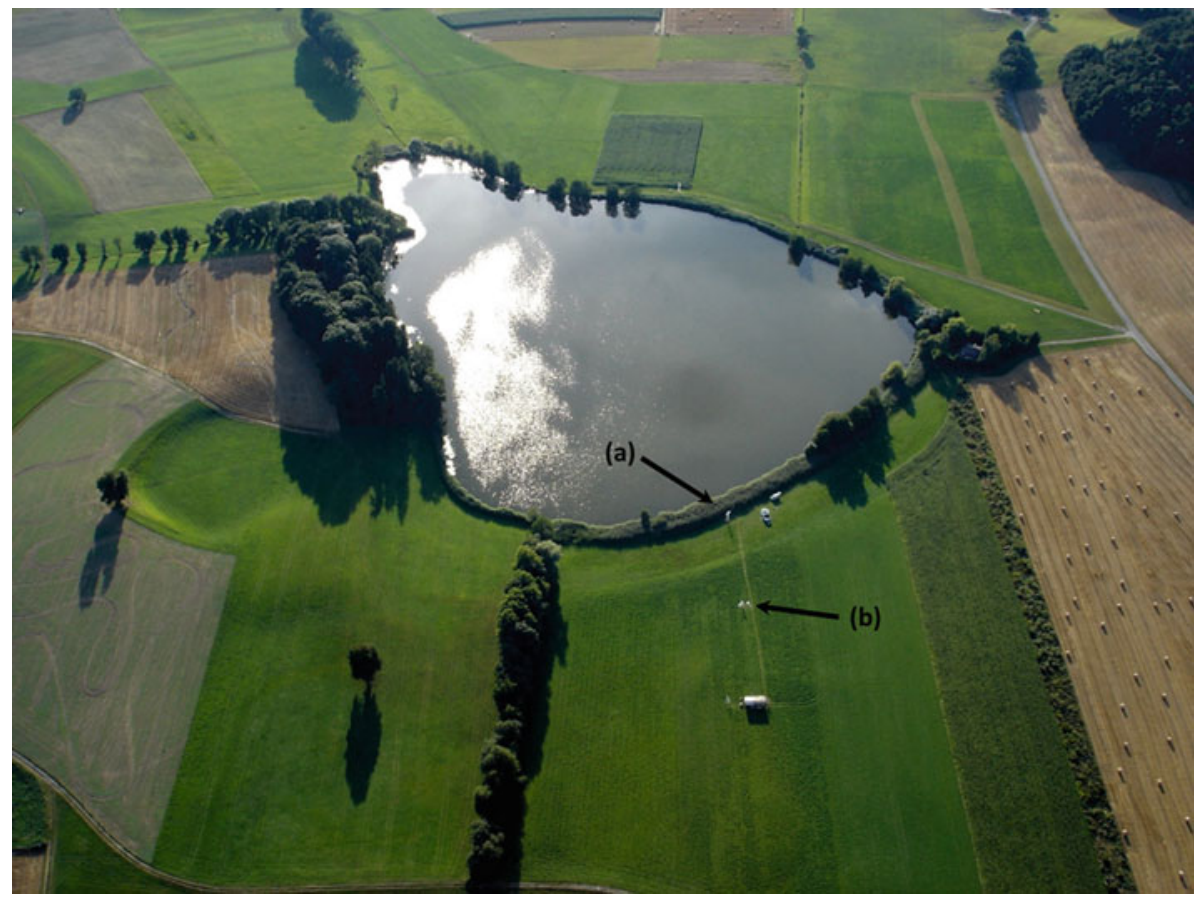

Fig. 1 Aerial photograph of the experimental site; the wind direction is computed using the two indicated sonic towers $(a)$ and $(b)$, and humidity measurements are taken between these two towers using the EPFL Raman lidar

\section{Field Measurement Campaign}

The TABLE experiment was performed in the summer of 2008 (from August 8 to September 5) near the town of Seedorf, Switzerland on the Swiss plateau. High spatial $(1.25 \mathrm{~m})$ and temporal (1s) resolution measurements of humidity over a water/land transition were performed by the EPFL Raman lidar. To calculate turbulent fluxes and to validate the Raman lidar measurements, three eddy-flux measurement stations were placed along the measurement transect. The average height of the lidar beam was $5.5 \mathrm{~m}$, while each eddy-flux station contained a CSAT3 three-dimensional sonic anemometer and a LI7500 fast response hygrometer. With each eddy-correlation set-up at 62, 129 and $490 \mathrm{~m}$ from the lidar, we have in situ validation of the humidity signal, a measurement of the mean flow velocity ( $U$ throughout the paper) and the wind direction. An aerial photograph of the experiential set-up is provided in Fig. 1. A more detailed description of the experimental set-up, statement of purpose, detailed accounting of the instrumentation, and description of the EPFL Raman lidar can be found in Froidevaux et al. (2012).

To control the quality of the humidity data, humidity fluctuations from both the Raman lidar and point measurements of the Licor 7500 hygrometers are directly compared. A time trace for both are presented in Fig. 2a and b for two of the hygrometers (62 and $129 \mathrm{~m}$ ) respectively. We see that the variance of the lidar data is comparable for tower 2 while being greater for tower 3 . The greater variance of the lidar humidity measurements relative to the Licor 7500 measurements at tower 2 is likely due to a decrease in signal-to-noise ratio of the lidar signal with increasing range. The time spectra of the lidar and Licor 7500 measurements are 

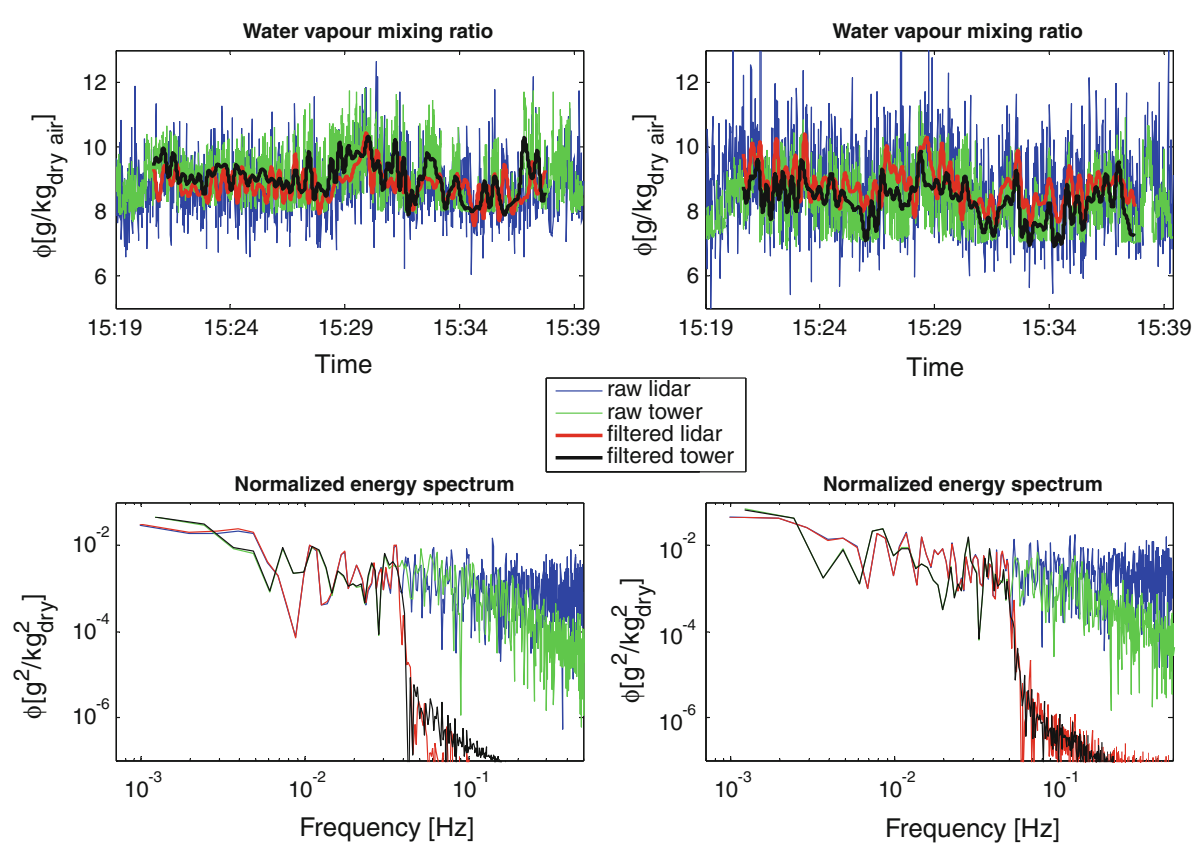

$$
\begin{aligned}
& \text { - raw lidar } \\
& \text { raw tower } \\
& \text { - filtered lidar } \\
& \text { - filtered tower }
\end{aligned}
$$

Normalized energy spectrum

Fig. 2 Time series and spectral density $(\phi)$ collocated Raman lidar and LI7500 humidity measurements. The data are spectrally filtered to eliminate the noise floor present in the lidar data, and to ensure that the mean and variance of the lidar measurements agree with the LI7500 measurements

presented in Fig. 2c and d. Spectra are computed with linearly detrended data using a standard Hanning window and the Matlab fast Fourier transform function. Here it is evident that the lidar spectra have a noise floor near $0.2 \mathrm{~s}^{-1}$. To then compare directly the two measurements, we filter both spectra with a low-pass filter of a characteristic length scale $\Delta$, thus eliminating the noise floor in the lidar while simultaneously eliminating the small-scale contributions to the variance in the Licor hygrometer. The resulting spectra are then retransformed to real space. These noise controlled data are also presented in Fig. $2 \mathrm{a}$ and $\mathrm{b}$ and we see that the variance is reduced and the traces track substantially closer to each other, although there are still noticeable differences in the signals. The filter operation is repeated to find the smallest filter time scale while ensuring the signal variances match. Recall that the sensors are not perfectly collocated, and the volume of the atmosphere being measured by the LI7500 and the EPFL Raman lidar is not identical. Twenty-min averages of humidity were also computed from the lidar and Licor 7500 time series; these averages were highly correlated throughout the field campaign (correlation coefficient of 0.97).

Water vapour alters the air density slightly (less than $1 \%$ for the current experimental conditions), but given the available dataset, humidity is treated as a passive tracer, and its measurements in space and time are used to compute the correlation function in Eq. 2. To ensure the best data quality, only the section from 20 to $140 \mathrm{~m}$ away from the lidar is used. Recall that the eddy-flux towers are located 62 and $129 \mathrm{~m}$ over the clover field from the light source respectively; therefore the chosen spatial extent for the humidity measurements has the added advantage of being bracketed by velocity measurements. The data are then broken into 2.5-min segments and the wind direction computed from the CSAT3 anemometers at the two previously mentioned tower locations. Data segments were selected such that the 
average wind direction (over $2.5 \mathrm{~min}$ ) was aligned within $2^{\circ}$ of the lidar measurement line. After two weeks of measurements, nine such segments were identified resulting in a total of $22.5 \mathrm{~min}$ of available data.

\section{Analysis of Field Measurements}

A space-time plot of the measured humidity is shown in Fig. 3. The diagonal pattern in this plot arises from large-scale $(50-100 \mathrm{~m})$ flow features that move with the mean flow and transect the lidar beam for 50-100 seconds; these long-lived structures are investigated in more detail in Froidevaux et al. (2012). A plot of the correlation function (Eq. 2) for one of the nine data segments is shown in Fig. 4. Here there is no correlation beyond the first few metres, and no evidence per se that Taylor's hypothesis can be supported beyond the resolution of the measurements. This is in contrast to the observed large advected structures present in Fig. 3, suggesting that the applicability of Taylor's hypothesis strongly depends on the length scale of interest (in this dataset the large scales are apparently more persistent).

To investigate the influence of scale on Taylor's hypothesis, the data are decomposed via Daubechies wavelet decomposition (Katul and Parlange 1995), and for each level of wavelet coefficients, we compute the correlation function (Eq. 2) using the resulting wavelet coefficients. The results (for a representative 2.5-min segment) for the lowest four levels of wavelet coefficients are shown in Fig. 5. Each set of wavelet coefficients represents a unique length scale (i.e. 2.5, 5, 10, 20 and 40m). In Fig. 5, the results are shown for length scale of $2.5 \mathrm{~m}$ (a), $5 \mathrm{~m}$ (b), $10 \mathrm{~m}$ (c), and $20 \mathrm{~m}$ (d). From Fig. 5 it is evident that, as the length scale of the turbulent structures grows, the time scale over which Taylor's hypothesis is applicable also grows.

Rescaling the abscissa by the average wind speed measured by the sonic anemometers, $U$, allows for all data segments to be averaged. This averaged result is shown in Fig. 6, where a one-to-one line is also included to indicate $\delta x / \delta t=U$, and where we would expect the maximum correlation at any distance. If we define a cut-off correlation where Taylor's hypothesis is no longer acceptable (20\%) and then solve for the intersection of the one-to-one line with the isoline of $20 \%$ correlation, we can directly read off the maximum distance (or scaled time) over which Taylor's hypothesis is acceptable for a given scale. A plot showing the relationship between this 'structure lifetime' and scale is presented in Fig. 7, where the scales are broken into two segments - those scales larger than the measurement height and those scales

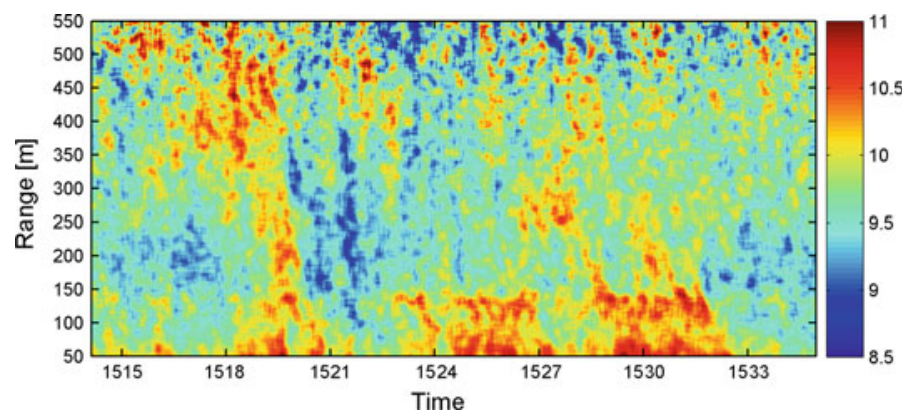

Fig. 3 An example plot of humidity measured in space and time with the EPFL Raman lidar. Humidity 'streaks' represent large-scale advected structures. Note that this image has been smoothed for presentation and to highlight the large-scale features of the flow 


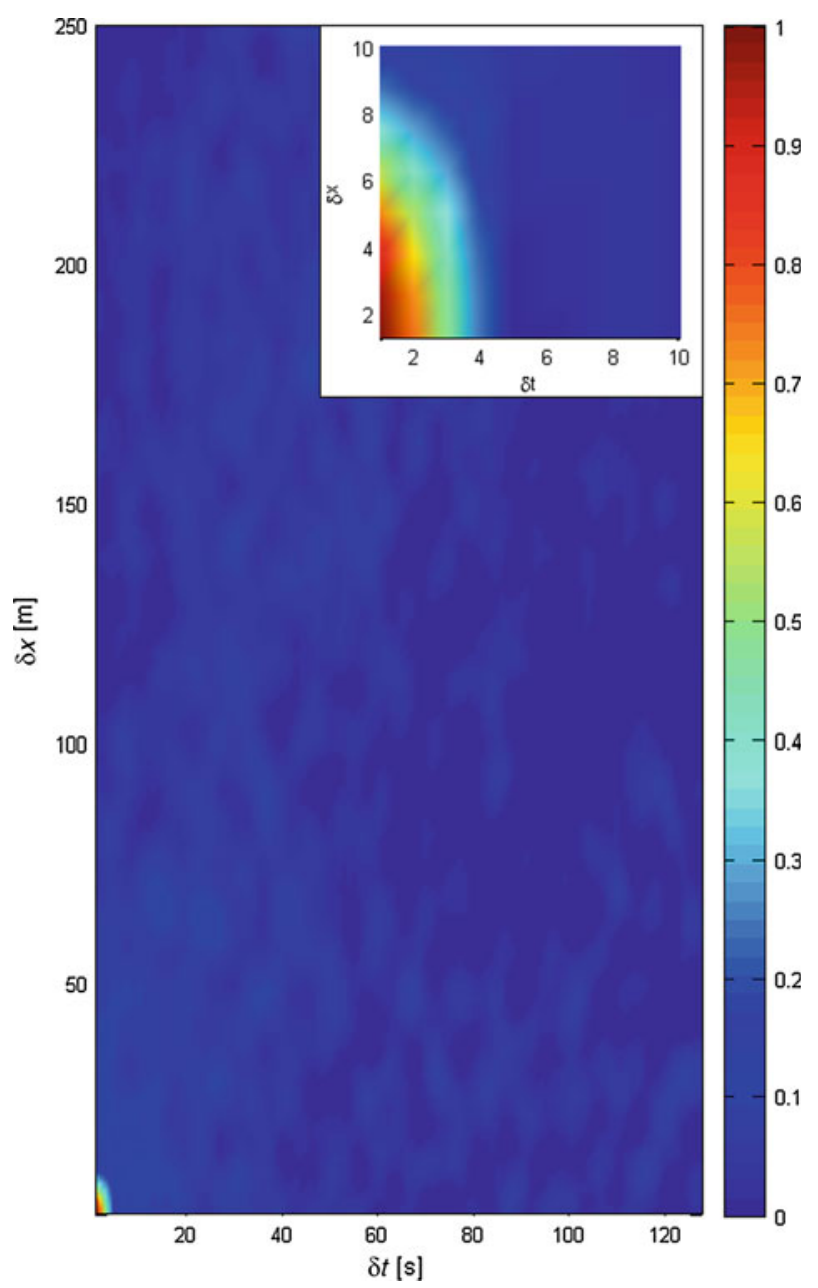

Fig. 4 The space-time correlation function, $\Phi(\delta x, \delta t)$, computed using the Raman lidar humidity data. Inset zoom of the section $0 \leq \delta \times \leq 10 \mathrm{~m}$ and $0 \leq \delta t \leq 10 \mathrm{~s}$, showing correlations $>0.8$. For all other values of $\delta x$ and $\delta t$, the correlation is $<0.15$

smaller than the measurement height. A range of correlation times are represented for some scales as there are multiple crossings in the data. In the plot, the minimum and maximum crossings are shown. Figure 7 shows that the applicability of Taylor's hypothesis for the field data follows a linear scaling relationship

$$
\frac{U t}{D} \sim p,
$$

where $U$ is the flow velocity measured with sonic anemometry, $D$ is the characteristic length scale of the turbulent structure, and $t$ is the 'lifetime' of the structure defined by when its autocorrelation falls below $20 \%$ along its Lagrangian path. For the present purpose we define the parameter, $p$, in Eq. 3 as the 'persistency scale', and it is clear from Fig. 7 that smaller scales have a lower persistency scale. 

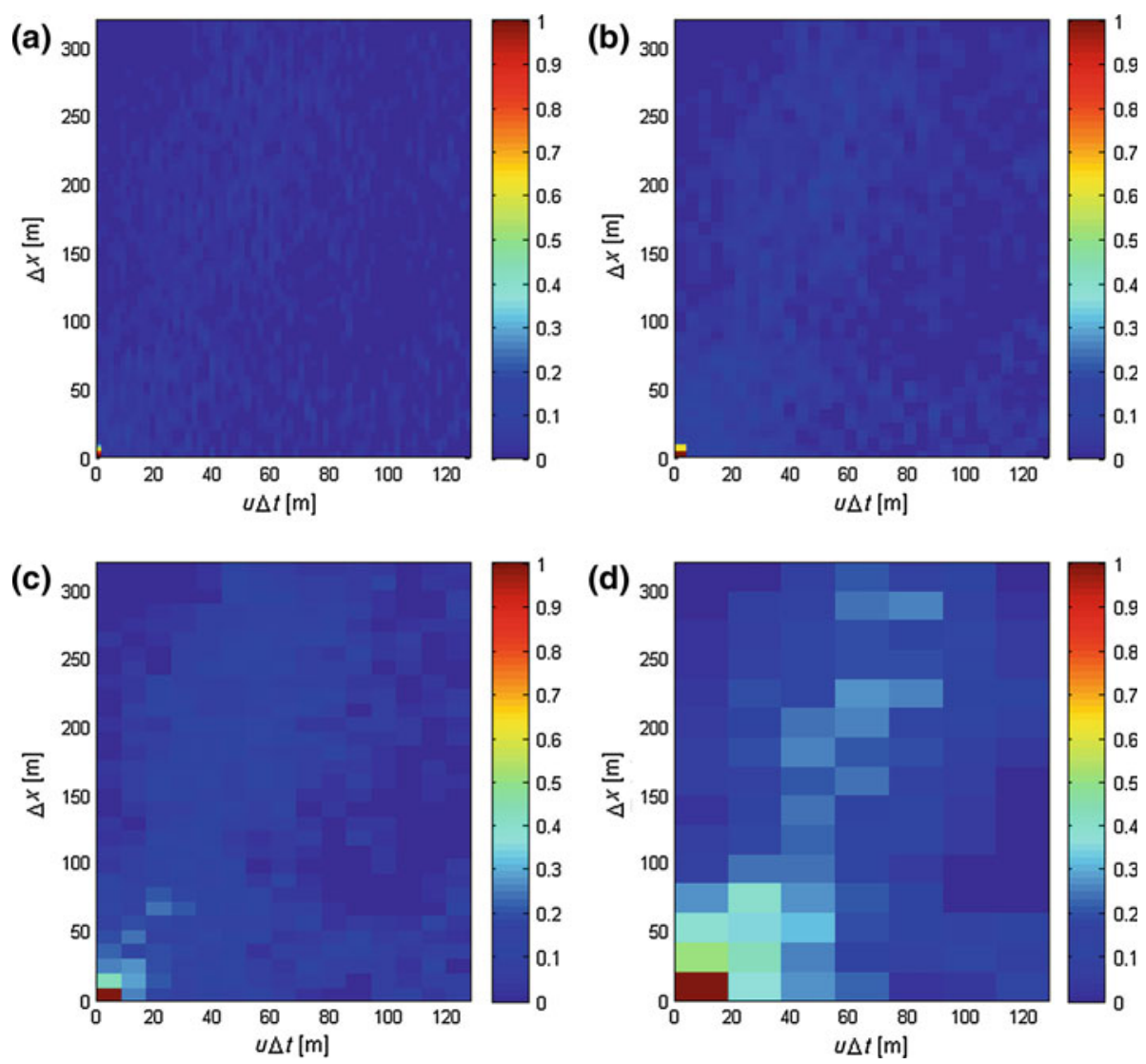

Fig. 5 The space-time correlation function computed at four different turbulence scales for a typical segment: a $2.55 \mathrm{~m}$, b $5 \mathrm{~m}, \mathbf{c} 10 \mathrm{~m}$, and $\mathbf{d} 20 \mathrm{~m}$. Here it is readily apparent that larger scales of motion are better suited to the use of Taylor's hypothesis

One can define a persistency scale for any correlation threshold, and we set the threshold at $20 \%$, though this choice is arbitrary. A plot of the average persistency scale versus correlation is shown in Fig. 8. For Taylor's hypothesis to be valid, the persistency scale must be greater than one since a turbulent structure must have a lifetime long enough for it to be completely sampled by a stationary instrument. As the autocorrelation criteria become more and more strict, the persistency scale diminishes until ultimately it crosses below unity. This crossing takes place at $33 \%$ correlation for the small scales, and at $61 \%$ for the large scales, implying that during the advection of the smaller turbulent structure across a hypothetical instrument each structure loses $67 \%$ of its original structure by the time it is fully sampled. For larger scales (greater than the measurement height) the result is more favourable with only $39 \%$ loss in correlation.

\section{Large-Eddy Simulation}

To cross check the analysis of the field measurements, LES of the lower atmosphere (see Deardorff 1974; Moeng 1984; Mason 1994; Albertson and Parlange 1999; Beare et al. 2006) 

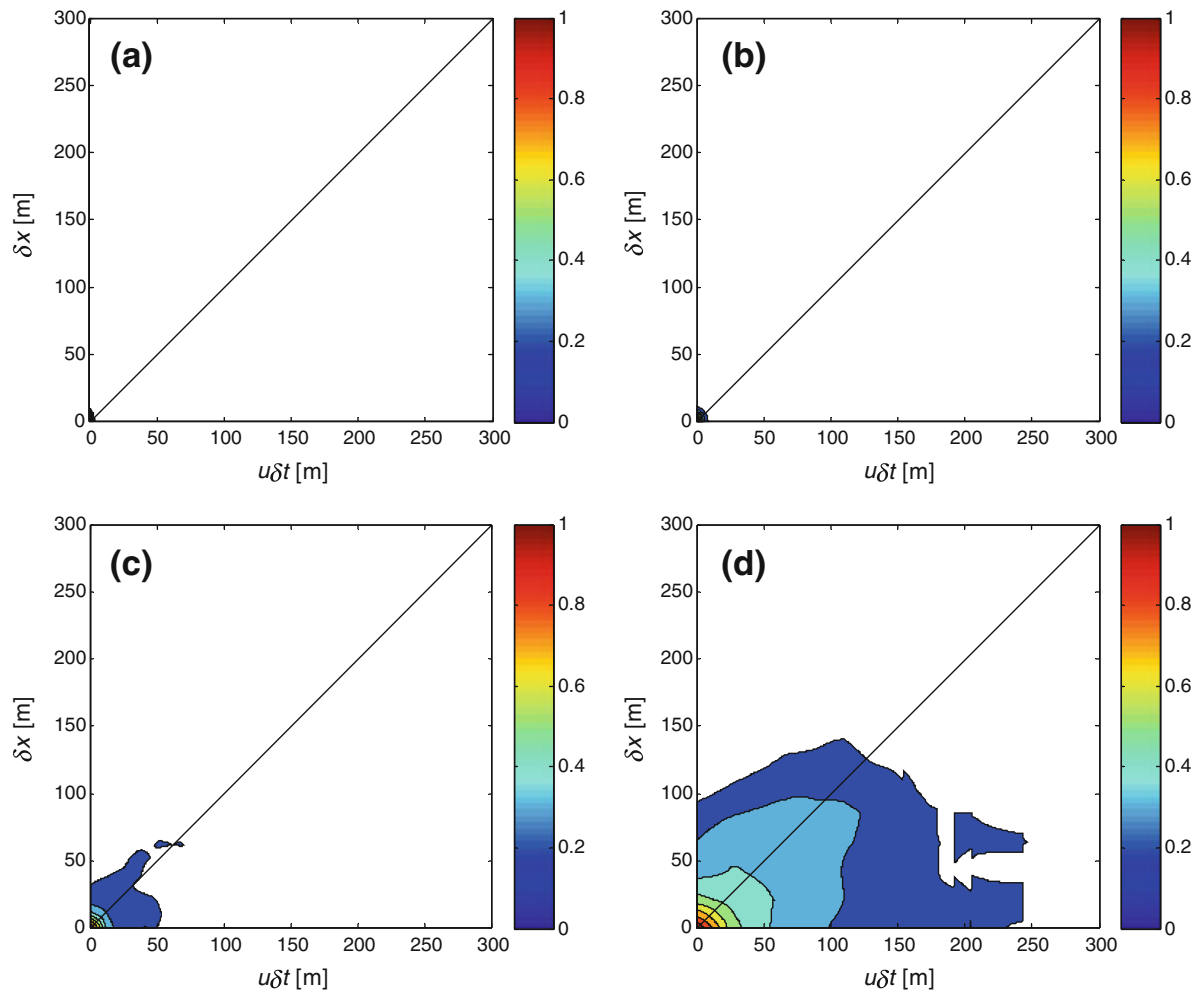

Fig. 6 Composite average of all data with a scaled time axis. The 1:1 line represents the advection velocity and expected correlation maxima for: a $2.5 \mathrm{~m}, \mathbf{b} 5 \mathrm{~m}, \mathbf{c} 10 \mathrm{~m}$, and $\mathbf{d} 20 \mathrm{~m}$

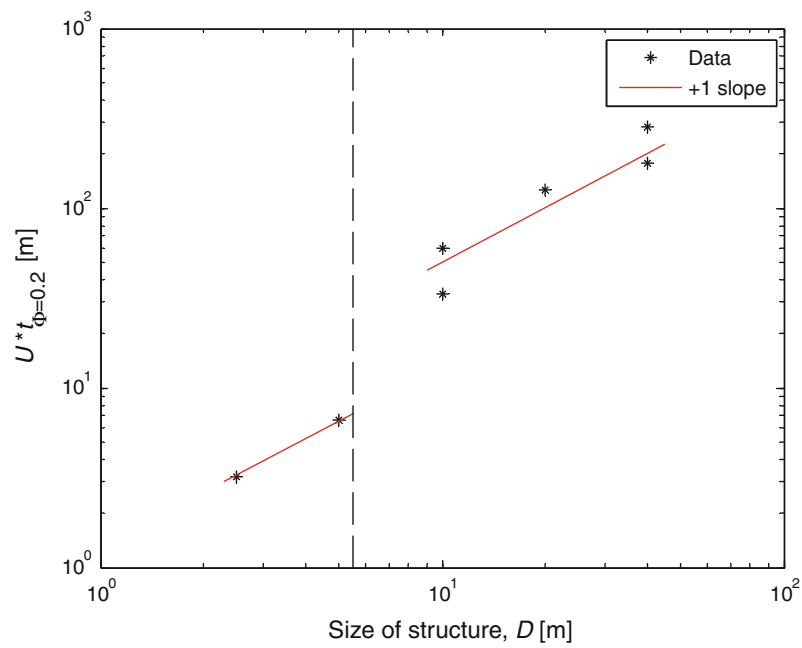

Fig. 7 Plot of the size of structure versus lifetime of structure as defined by an $80 \%$ correlation loss. This formulation indicates a scaling slope of unity, consistent with a Buckingham Pi dimensional analysis approach. Note that the ratio $U t / D$ is much larger for structures larger than the measurement height (indicated by the dashed line) 


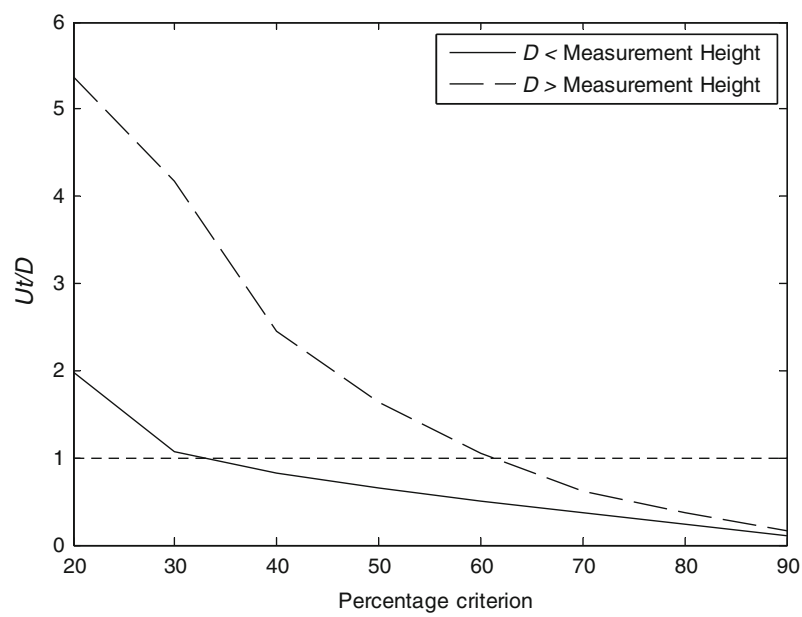

Fig. 8 The persistency as a function of correlation for both small-scale and large-scale structures. When the persistency drops below 1, a single eddy can no longer be resolved by a stationary sensor. Therefore the crossing of 1 indicates the optimal acceptance cut-off for the applicability of Taylor's hypothesis for those scales of motion

were also performed. LES is a numerical tool particularly well suited for this comparison since it is capable of resolving turbulent fluid motions on temporal and spatial scales comparable to the measurements. LES simulates the transport of mass, momentum and energy in the atmospheric boundary layer using the filtered incompressible Navier-Stokes equation together with the filtered scalar conservation equations (Tennekes and Lumley 1972; Pope 2000). The initial conditions are set by an approximation of measured temperature and specific humidity profiles. The simulated domain is $1024 \times 512 \mathrm{~m}^{2}$ in the horizontal directions and $256 \mathrm{~m}$ in the vertical, while the numerical resolution used is $512 \times 256 \times 128$ nodes, leading to a spatial resolution of $2 \mathrm{~m}$ in all directions, with the first vertical node at $1 \mathrm{~m}$. The bottom boundary is fixed at a constant temperature $(292 \mathrm{~K})$ and a constant mixing ratio $\left(13 \mathrm{~g} \mathrm{~kg}^{-1}\right)$, with the lower boundary conditions enforced through a local implementation of Monin-Obukhov similarity described in Bou-Zeid et al. (2005). The flow is forced with a mean pressure gradient aligned with the mean flow direction. The subgrid-scale model is the Lagrangian scale dependent dynamic model (Bou-Zeid et al. 2005).

The full analysis above is repeated using data from the LES. Here a single line of data at a height of $10 \mathrm{~m}$ above the simulation surface is extracted for analysis, and for consistency we also use humidity as a proxy to test Taylor's hypothesis. The full computed correlation function is plotted in Fig. 9. It is evident that, for the simulations, Taylor's hypothesis is more applicable. This is due in part to the prescribed, perfectly oriented, mean flow. In the data, although we are very strict in our definition of acceptable wind directions, there are small meandering motions present in all data segments, in addition to measurement noise.

When the simulation results are decomposed into wavelet coefficients and the previous analysis repeated for the LES data (Fig. 10), it is clear that larger scales still have a greater persistency than the smaller scales; however the smallest scales are far more persistent in the LES than they were in the data. Finally, the persistency scale as a function of correlation is presented in Fig. 11 as an average for all scales. We do not separate scales in this case as they all have the same behaviour, and is likely due to the fact of the finite domain size of the LES, and thus the restriction on the formation of very large scales. The point at which Taylor's 


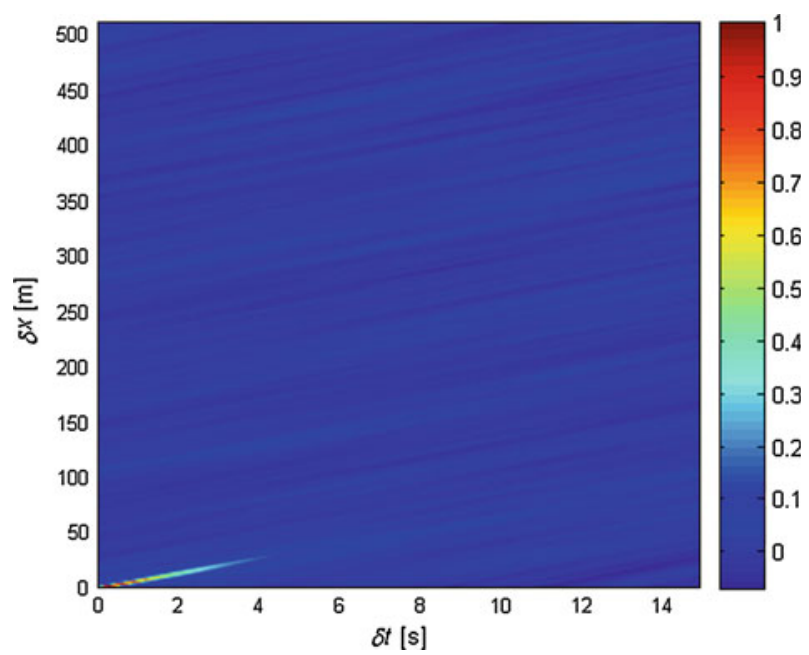

Fig. 9 The space-time correlation function of the LES data. Note that here there is a much higher correlation over a longer time (compared to the field data)
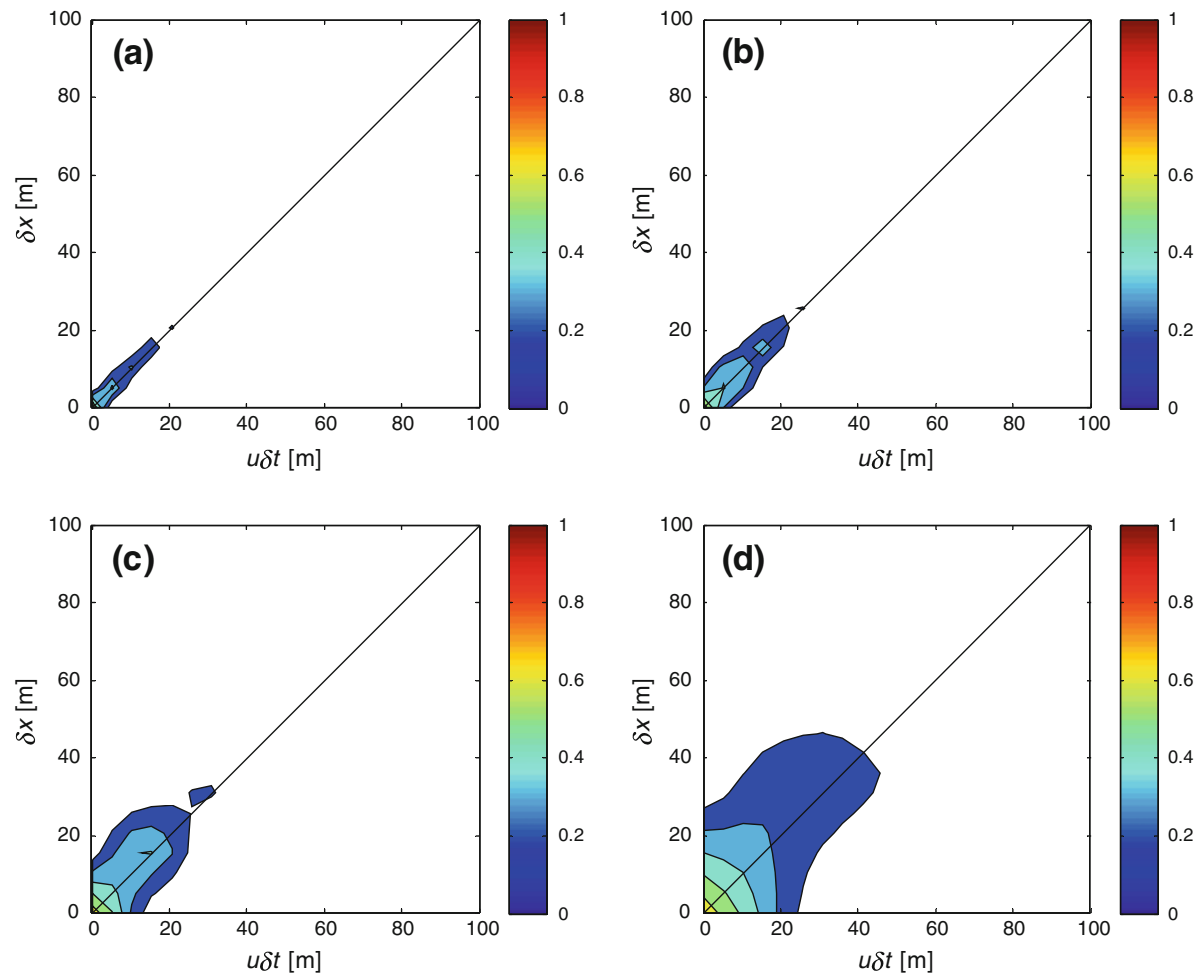

Fig. 10 The space-time correlations on wavelet coefficients for the LES data for the scales a $4 \mathrm{~m}, \mathbf{b} 8 \mathrm{~m}$, c $16 \mathrm{~m}$, and $\mathbf{d} 32 \mathrm{~m}$. Here the smallest scales have a much better behaviour with respect to Taylor's hypothesis 


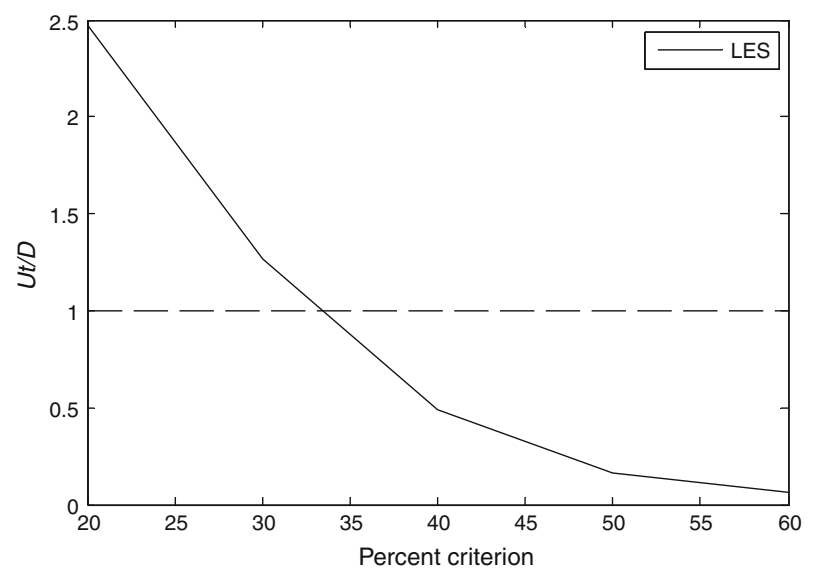

Fig. 11 The persistency as a function of correlation for the LES data. As we are limited by the domain size, the LES is only able to simulate scales consistent with the 'small scales' of the field experiment. Here the crossing of the $u t / D=1$ line is consistent with the field measurements at around $34 \%$

hypothesis will no longer hold is a $34 \%$ correlation, which is in close agreement with the field measurements.

\section{Conclusions}

The applicability of Taylor's frozen turbulence hypothesis is strongly dependent on the scale of the turbulent structures that are investigated. Atmospheric structures larger than the measurement height tend to be especially long lived and are the best candidates for the applicability of Taylor's hypothesis from the analysis of our field data. Turbulent structures smaller than the measurement height are less persistent, and thus Taylor's hypothesis must be applied in a stricter way (i.e. the acceptable correlation cut-off must be reduced). Both simulations and experimental data agree that this cut-off is $\approx 33-34 \%$, implying that a single eddy may change significantly while it is resolved by a stationary sensor. Larger flow features retain a higher degree of autocorrelation. Figure 8 provides a framework to determine the applicability of Taylor's hypothesis given the length and time scales of interest.

Acknowledgments The authors would like to thank the Swiss National Science Foundation (project no. 200021-107910/1, 200021_120238/1, 2008-2011) and the Mobile Information Communication System (MICS) for their support of this research.

Open Access This article is distributed under the terms of the Creative Commons Attribution License which permits any use, distribution, and reproduction in any medium, provided the original author(s) and the source are credited.

\section{References}

Albertson JD, Parlange MB (1999) Natural integration of scalar fluxes from complex terrain. Adv Water Res 23(3):239-252

Bahraminasab A, Niry MD, Davoudi J, Tabar MRR, Masoudi AA, Sreenivassan KR (2008) Taylor's frozenflow hypothesis in Bergers turbulence. Phys Rev E 77(6):Art No. 065302 
Beare RJ, MacVean MK, Holtslag AAM, Cuxart J, Esau I, Golaz JC, Jimenez MA, Khairoutdinov M, Kosovic B, Lewellen D, Lund TS, Lundquist JK, McCabe A, Moene AF, Noh Y, Raasch S, Sullivan P (2006) An intercomparison of large-eddy simulations of the stable boundary layer. Boundary-Layer Meteorol 118(2):247-272

Bou-Zeid E, Meneveau C, Parlange MB (2005) A scale-dependent Lagrangian dynamic model for large eddy simulation of complex turbulent flows. Phys Fluids 17:025105-1

Bou-Zeid E, Higgins CW, Huwald H, Meneveau C, Parlange MB (2010) Field study of the dynamics and modeling of subgrid-scale turbulence in a stable atmospheric surface layer over a glacier. J Fluid Mech 665:80-515

Dahm WJA, Southerland KB (1997) Experimental assessment of Taylor's hypothesis and its applicability to dissipation estimates in turbulent flows. Phys Fluids 9(7):2101-2107

Deardorff JW (1974) Three-dimensional numerical study of the height and mean structure of a heated planetary boundary layer. Boundary-Layer Meteorol 7(1):81-106

Del Alamo JC, Jimenez J (2009) Estimation of turbulent convection velocities and corrections to Taylor's approximation. J Fluid Mech 640:5-26

Dosio A, De Arellano JVG, Holtslag AAM, Builtjes PJH (2005) Relating Eularian and Lagrangian statistics for the turbulent dispersion in the atmospheric convective boundary layer. J Atmos Sci 62(4):1175-1191

Froidevaux M, Higgins CW, Simeonov V, Ristori P, Pardyjak E, Serikov I, Parlange MB (2012) A new Raman lidar to measure water vapor in the atmospheric boundary layer. Adv Water Res (under review)

Higgins CW, Parlange MB, Meneveau C (2003) Alignment trends of velocity gradients and subgrid-scale fluxes in the turbulent atmospheric boundary layer. Boundary-Layer Meteorol 109(1):59-83

Higgins CW, Parlange MB, Meneveau C (2004) The heat flux and the temperature gradient in the lower atmosphere. Geophys Res Lett 31(22):Art. No. L22105

Higgins CW, Meneveau C, Parlange MB (2009) Geometric alignments of the subgrid-scale force in the atmospheric boundary layer. Boundary-Layer Meteorol 132:1-9

Hill RJ (1996) Corrections to Taylor's frozen turbulence approximation. Atmos Res 40:153-175

Horst TW, Kleissl J, Lenschow DH, Meneveau C, Moeng C-H, Parlange MB, Sullivan PP, Weil JC (2004) HATS, field observations to obtain spatially-filtered turbulence fields from transverse arrays of sonic anemometers in the atmospheric surface layer. J Atmos Sci 61(13):1566-1581

Katul GG, Parlange MB (1995) The spatial structure of turbulence at production wave-numbers using orthonormal wavelet. Boundary-Layer Meteorol 75(1-2):81-108

Kelly M, Wyngaard JC, Sullivan PP (2009) Application of a subfilter scale flux model over the ocean using OHATS field data. J Atmos Sci 66:3217-3225

Kleissl J, Meneveau C, Parlange MB (2003) On the magnitude and variability of subgrid-scale eddy-diffusion coefficients in the atmospheric boundary layer. J Atmos Sci 60(19):2372-2388

Kleissl J, Parlange MB, Meneveau C (2004) Field experimental study of dynamic Smagorinsky models in the atmospheric surface layer. J Atmos Sci 61(18):2296-2307

Lumley JL (1965) Interpretation of time spectra measured in high-intensity shear flows. Phys Fluids 8:10561062

Mason PJ (1994) Large eddy simulation-a critical review of the technique. Q J Roy Meteorol Soc 120(515):1-26

Moeng C-H (1984) A large-eddy simulation model for the study of planetary boundary-layer turbulence. J Atmos Sci 41:2052-2062

Moin P (2009) Revisiting Taylor's hypothesis. J Fluid Mech 640:1-4

Patton N, Horst ET, Sullivan P, Lenschow D, Oncley S, Brown W, Burns S, Guenther A, Held A, Karl T, Mayor S, Rizzo L, Spuler S, Sun J, Turnipseed A, Allwine E, Edburg S, Lamb B, Avissar R, Calhoun R, Kleissl J, Massman W, Paw-U K, Weil J (2011) The canopy horizontal array turbulence study (CHATS). Bull Am Meteoreol Soc 92:593-611

Pope SB (2000) Turbulent flows. Cambridge University Press, Cambridge, UK 806 pp

Porté-Agel F, Meneveau C, Parlange MB (1998) Some basic properties of the surrogate subgrid-scale heat flux in the atmospheric boundary layer. Boundary-Layer Meterol 88(3):425-444

Porté-Agel F, Meneveau C, Parlange MB (2000a) A scale dependent dynamic model for large eddy simulation: application to a neutral atmospheric boundary layer. J Fluid Mech 415:261-284

Porté-Agel F, Parlange MB, Meneveau C, Eichinger WE, Pahlow M (2000b) Subgrid-scale dissipation in the atmospheric surface layer: effects of stability and filter dimension. J Hydrometerol 1:75-87

Porté-Agel F, Parlange MB, Meneveau C, Eichinger WE (2001a) A priori field study of the subgrid-scale heat fluxes and dissipation in the atmospheric surface layer. J Atmos Sci 58(18):2673-2697

Porté-Agel F, Pahlow M, Meneveau C, Parlange MB (2001b) Atmospheric stability effect on subgrid scale physics for large-eddy simulation. Adv Water Resour 24(9-10):1085-1102

Taylor GI (1938) The spectrum of turbulence. Proc Roy Soc Lond 164:476-490 
Tennekes H, Lumley JL (1972) A first course in turbulence. MIT Press, Cambridge, USA, 300 pp

Thomas CK (2011) Variability of sub-canopy flow, temperature, and horizontal advection in moderately complex terrain. Boundary-Layer Meteorol 139:61-81

Tong CN (1996) Taylor's hypothesis and two point coherence measurements. Boundary-Layer Meteorol 81(3-4):399-410

Tong CN, Wyngaard JC, Brasseur JG (1999) Experimental study of the subgrid-scale stress in the atmospheric surface layer. J Atmos Sci 56(14):2277-2292

Willis GE, Deardorff JW (1976) On the use of Taylor's translation hypothesis for diffusion in the mixed layer. Q J Roy Meteorol Soc 102:817-822

Wyngaard JC, Clifford SF (1977) Taylor's hypothesis and high frequency turbulence spectra. J Atmos Sci 34:922-929 